\title{
Integral Resonant Control for vibration damping and precise tip-positioning of a single-link flexible manipulator
}

\author{
Emiliano Pereira, Member, IEEE, Sumeet S. Aphale, Member, IEEE, Vicente Feliu, Senior Member, IEEE \\ and S. O. Reza Moheimani, Senior Member, IEEE
}

\begin{abstract}
In this work we propose a control design method for single-link flexible manipulators. The proposed technique is based on the Integral Resonant Control (IRC) scheme. The controller consists of two nested feedback loops. The inner loop controls the joint angle and makes the system robust to joint friction. The outer loop, which is based on the IRC technique, damps the vibration and makes the system robust to the unmodeled dynamics (spill-over) and resonance frequency variations due to changes in the payload. The objectives of this work are: (i) to demonstrate the advantages of IRC, which is a high performance controller design methodology for flexible structures with collocated actuator-sensor pairs, and (ii) to illustrate its capability of achieving precise end-point (tip) positioning with effective vibration suppression when applied to a typical flexible manipulator. The theoretical formulation of the proposed control scheme, a detailed stability analysis and experimental results obtained on a flexible manipulator are presented.
\end{abstract}

Index Terms-Flexible manipulators, Precise tip positioning, Vibration damping, Integral Resonant Control

\section{INTRODUCTION}

The past two decades have seen significant interest in the control of flexible robotic manipulators. Novel robotic applications demand lighter robots that can be driven using small amounts of energy. An example of this is robotic booms in the aerospace industry, where lightweight robot manipulators with high performance requirements (high speed operation, better accuracy) are demanded [1]. Unfortunately, the flexibility of these robotic arms leads to oscillatory behaviour at the tip of the link, making precise pointing or tip positioning a daunting task that requires complex closed-loop control. In order to address control objectives, such as tip position accuracy and suppression of residual vibration, many control techniques have been applied to flexible robots [2], [3], [4].

There are two main problems that complicate the control design for flexible manipulators viz: (i) the high order of the system and (ii) the non-minimum phase dynamics that exist between the tip position and the input torque applied at the joint. These problems have motivated researchers to employ a wide range of control techniques such as Linear Quadratic Gaussian (LQG) [5], Linear Quadratic Regulator (LQR) [6], pole placement [7], and inverse dynamics based control [8]. The drawback of these model-based approaches is that the complexity of the control algorithm increases significantly with the system order and the stability of the closed-loop system is sensitive to (a) changes in the robot payload, (b) model parameter uncertainties and (c) high-order unmodeled dynamics since a wide control bandwidth may lead to spill-over effect [9]. In order to address these problems, solutions based on adaptive control [10], $H_{\infty}$ control [11], $\mu$-synthesis [12], sliding-mode control [13], neural networks [14] and fuzzy logic algorithms [15] have been investigated. However, the mathematical complexity and difficulties in their implementation make their application to flexible robotics less attractive.

The use of alternative outputs has emerged as a potential solution to the problem of non-minimum phase dynamics. In [16], an alternative output, the so-called reflected tip position, was proposed. It was demonstrated therein that the transfer function from the motor torque to the reflected tip velocity is passive. Therefore, strictly passive controllers make the system stable in $L_{2}$ sense. However, the passive relationship depends on the value of the hub inertia, which must be sufficiently small in relation with beam inertia. In [17], an additional control loop to make the passive relationship independent of the system parameters was proposed. However, the main limitation of these passivity based control schemes is that they make the system very sensitive to joint friction. Other solutions based on two control loops can be found in [18], [19] and [20]. The inner loop based on a collocated inputoutput arrangement, is used to damp the vibrations whereas the outer loop is used for position control. However, in [18] and [19], the stability of the position control depends on the link and motor parameters, which complicates the design. Although the vibration damping proposed in [20] was quite efficient when a motor without reduction gear was used, the residual vibration suppression is not effective when reduction gears are employed.

Recently, a novel control design scheme based on two control loops was proposed in [21] to make the system stability independent of the link and motor parameters. The difference in this control scheme when compared with the philosophy of the control schemes proposed in [18], [19] and [20] is that the outer loop is used to damp the vibrations and the inner loop is used for position control. The position controller only depends on the motor dynamics, and the outer loop always guarantees an effective vibration suppression (with or without the reduction gear). In addition, the stability analysis is simple and can be made independent of the link dynamics. However, the proposed outer loop controller has some disadvantages like high sensitivity at low frequencies that complicates its implementation on a practical experimental platform (offset 
in the sensors, gravity effect). It also suffers from instability issues due to anti-aliasing filters.

This work builds on the control methodology of [21] and proposes a new approach based on the Integral Resonant Control (IRC) scheme [22]. This methodology consists of: i) designing a joint position controller robust to Coulomb friction and payload variations, thus allowing accurate positioning of the joint; and ii) designing an outer controller that imparts substantial damping to link dynamics. This combined scheme results in an easy-to-tune, low-order damping controller that damps multiple resonant modes, without instability issues due to unmodeled system dynamics. This work substitutes the outer controller used in [21] by a modification of an IRC scheme, which retains the advantages of [21] and alleviates the aforementioned problems.

This paper is organized as follows. Section II provides a description of the experimental setup. The system model and the associated parameters are briefly explained in Section III. Section IV explains the proposed control scheme and also demonstrates the closed-loop stability. A practical implementation of the proposed control scheme on a singlelink flexible robot and the obtained experimental results are presented in Section V. This section also gives a detailed comparison of positioning performance achieved by various previously proposed and popular control schemes. Section VI concludes the paper.

\section{EXPERIMENTAL SETUP}

Figure 1 shows a photograph of the single-link flexible manipulator used as the experimental platform in this work. The setup consists of (a) a DC motor (Maxon Motor EC60) with a Harmonic Drive reduction gear 1:50 (HFUC-3250-20H), (b) a flexible single-link comprised of a slender aluminium beam with a rectangular cross section that is attached to the motor hub in such a way that it rotates only in the horizontal plane, so that the effect of gravity can be ignored and (c) a mass with negligible inertia at the end of the arm. In addition, two sensors are used: 1) an encoder mounted at the joint of the manipulator to measure the motor angle, and 2) a strain-gauge bridge placed at the base of the beam to measure the coupling torque. The physical parameters of the system are given in Table I. The strain signal is amplified by the dynamic strain amplifier (Kyowa DPM600) and filtered by a second-order Butterworth filter with its cut-off frequency set to $300 \mathrm{~Hz}$. A National Instruments 6024E and a Measurement Computing CIO-DIO24 are used in a $\mathrm{PC}$ in conjunction with the Real Time Windows Target of MATLAB. The sampling time is set to $0.002 \mathrm{~s}$. Finally, a dual channel Agilent-35670A spectrum analyzer was used for determining the frequency response functions (FRF's).

\section{SYSTEM MODEL}

Consider a flexible arm (see Figure 2) composed of: (a) a motor and a reduction gear of $1: n$ at the base with total inertia (rotor and hub) $J_{0}$, dynamic friction coefficient $\nu$ and Coulomb friction torque $\Gamma_{f}$; (b) a flexible beam with uniform linear mass density $\rho$, uniform bending stiffness $E I$ and length

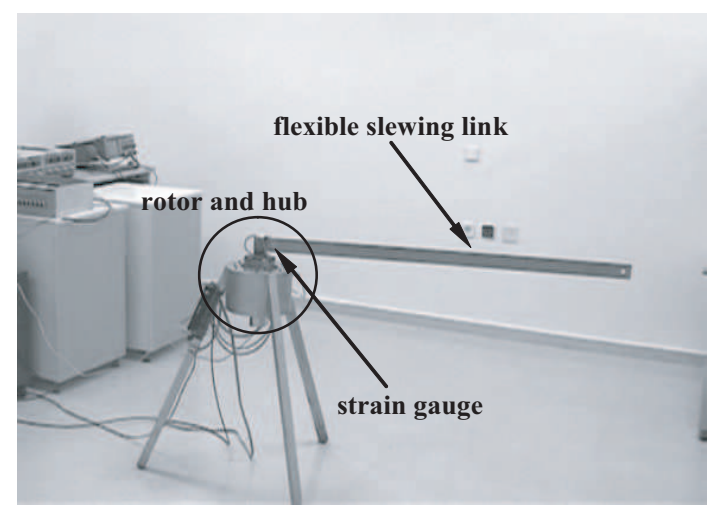

Fig. 1. Experimental platform.

\begin{tabular}{ccc}
\hline Stiffness $\left(N \mathrm{~m}^{2}\right)$ & $E I$ & 2.40 \\
\hline Width $(\mathrm{m})$ & $h$ & 0.05 \\
\hline Thickness $(\mathrm{m})$ & $b$ & 0.002 \\
\hline Length $(\mathrm{m})$ & $L$ & 1.26 \\
\hline Linear mass density $(\mathrm{kg} / \mathrm{m})$ & $\rho$ & 0.268 \\
\hline Mass of the beam $(\mathrm{kg})$ & $M_{b}$ & 0.338 \\
\hline Range of the payload mass $(\mathrm{kg})$ & $M_{P}$ & $0.0-0.300$ \\
\hline Motor constant $(N \mathrm{~N} / \mathrm{V})$ & $K$ & 0.474 \\
\hline Reduction gear ratio & $n$ & 50 \\
\hline Inertia of the motor and hub $\left(\mathrm{kgm}^{2}\right)$ & $J_{0}$ & 0.79
\end{tabular}

PARAMETERS OF THE FLEXIBLE ARM

$L$; and (c) a payload of mass $M_{P}$ and rotational inertia $J_{P}$. Furthermore, the applied torque is $\Gamma_{m}, w(x, t)$ is the elastic deflection measured from the undeformed beam, $\theta_{m}$ is the joint angle and $\theta_{t}$ is the tip angle. Note that parametric representation of Figure 2 corresponds with the so call pseudoclamped configuration, in which the non-inertial frame $(x, y)$ rotates with the motor and the overall structure rotates in an inertial frame $(\mathrm{X}, \mathrm{Y})$. Using the variational calculus as [23], the next boundary value problem can be defined

$$
\begin{array}{r}
E I w^{\prime \prime \prime \prime}(x, t)+\rho\left(x \ddot{\theta}_{m}(t)+\ddot{w}(x, t)\right)=0, \\
\Gamma_{m}(t)=n K V(t)=J_{0} \ddot{\theta}_{m}(t)+\nu \dot{\theta}_{m}(t)+\Gamma_{\text {coup }}(t)+\Gamma_{f}(t),
\end{array}
$$

in which the (') and (') indicates derivation with respects to $t$ and $x, V$ is a voltage that controls the motor; $K$ is a constant that relates the motor torque $\left(\Gamma_{m}\right)$ and the control voltage $(V)$; and $\Gamma_{\text {coup }}$ is the coupling torque in the joint due to the link and the payload, and can be obtained using the formula:

$$
\Gamma_{\text {coup }}(t)=-E I w^{\prime \prime}(0, t),
$$

where $w^{\prime \prime}(0, t)$ is proportional to the strain measured at the base of the link. The boundary conditions are

$$
\begin{array}{r}
w(0, t)=0, w^{\prime}(0, t)=0, \\
E I w^{\prime \prime}(L, t)=-J_{P}\left(\ddot{\theta}_{m}(t)+\ddot{w}^{\prime}(L, t)\right),
\end{array}
$$




$$
E I w^{\prime \prime \prime}(L, t)=M_{P}\left(L \ddot{\theta}_{m}(t)+\ddot{w}(L, t)\right) .
$$

The boundary problem is solved by using separability of the variables and modal expansion. Thus, the elastic deflection is assumed as follows

$$
w(x, t)=\sum_{i=1}^{\infty} \phi_{i}(x) \delta_{i}(t)
$$

being $\phi_{i}$ and $\delta_{i}$ spatial and time solution of Eq. (1) for the $i$ vibration mode. Thus, proceeding in the same way as described in [24], the model between $w(x, t)$ (or any function of $w(x, t))$ and $\Gamma_{m}$ can be obtained if $\Gamma_{f}$ is not considered in model deduction. If $w^{\prime \prime}(x, t)$ is calculated from Eq. (5) and it is substituted into (3), $\Gamma_{\text {coup }}$ is as following

$$
\Gamma_{\text {coup }}(t)=-E I \sum_{i=1}^{\infty} \phi_{i}^{\prime \prime}(0) \delta_{i}(t)
$$

It can be proved that $\phi_{i}^{\prime \prime}(0) \neq 0 \forall i$, which guarantees that the link vibration is zero if and only if $\Gamma_{\text {coup }}(t)=0$. In addition, $\Gamma_{\text {coup }}$ is also the result of the reaction torque in the link due to the joint acceleration and link vibrations, which makes $\Gamma_{\text {coup }}(s) / s \theta_{m}(s)$ a passive system [21]. If $\Gamma_{f}$ is neglected in Eq. (2), the transfer function between $\theta_{m}(s)$ and $\Gamma_{\text {coup }}(s)$ can be expressed as follows

$$
\frac{\theta_{m}(s)}{\Gamma_{m}(s)}=\frac{\left(n K / J_{0}\right) /\left(s\left(s+\nu / J_{0}\right)\right)}{1+\left(\left(n K / J_{0}\right) /\left(s\left(s+\nu / J_{0}\right)\right)\right)\left(\Gamma_{\text {coup }}(s) / \theta_{m}(s)\right)},
$$

which is used to obtain the expression of $\Gamma_{\text {coup }}(s) / \theta_{m}(s)$. It must be noted that the zeros of $\theta_{m}(s) / \Gamma_{m}(s)$ are the poles of $\left(\left(n K / J_{0}\right) /\left(s\left(s+\nu / J_{0}\right)\right)\right)\left(\Gamma_{\text {coup }}(s) / \theta_{m}(s)\right)$. Then, the zeros of $\theta_{m}(s) / \Gamma_{\text {coup }}(s)$ are obtained by using the poles of $\theta_{m}(s) / \Gamma_{m}(s)$ and the denominator of Eq. (7). Thus, the resulting expression of the system $\Gamma_{\text {coup }}(s) / \theta_{m}(s)$, which is deduced by assuming a link with distributed mass, is as follows

$$
\frac{\Gamma_{\text {coup }}(s)}{\theta_{m}(s)}=\left(\frac{\Gamma_{m}(0)}{\theta_{m}(0)}\right) \sum_{i=1}^{N} \frac{s^{2} / z_{i}^{2}+1}{s^{2} / \omega_{i}^{2}+1}
$$

where $N$ the number of considered vibration modes, $z_{i}$ and $\omega_{i}$ the zeros and poles of $\Gamma_{\text {coup }}(s) / \theta_{m}(s)$ respectively. Note that the term $\ddot{\theta}_{m}$ in Eq. (1) involves two zeros in the origin in Eq. (8) (i.e., $z_{1}=0$ ). Finally, the tip angle, which can be defined as follows

$$
\theta_{t}(t)=\theta_{m}(t)+w(L, t)
$$

is accurately positioned to its desired position $\theta_{t}^{*}$ if $\theta_{m} \equiv \theta_{t}^{*}$ and $w(L, t)=0$ for $t \geq t_{s}$, being $t_{s}$ the settling time. The condition $w(L, t)=0$ is achieved in this work by actively damping $\theta_{m}(s) / \Gamma_{\text {coup }}(s)$.

\section{Proposed CONTROL SCHEME}

The proposed control methodology accurately positions $\theta_{m}$ and actively damps link vibrations to achieve a precise tip positioning by an inner and an outer control loops respectively. This section describes the utilized controllers and demonstrates the stability of overall control scheme.

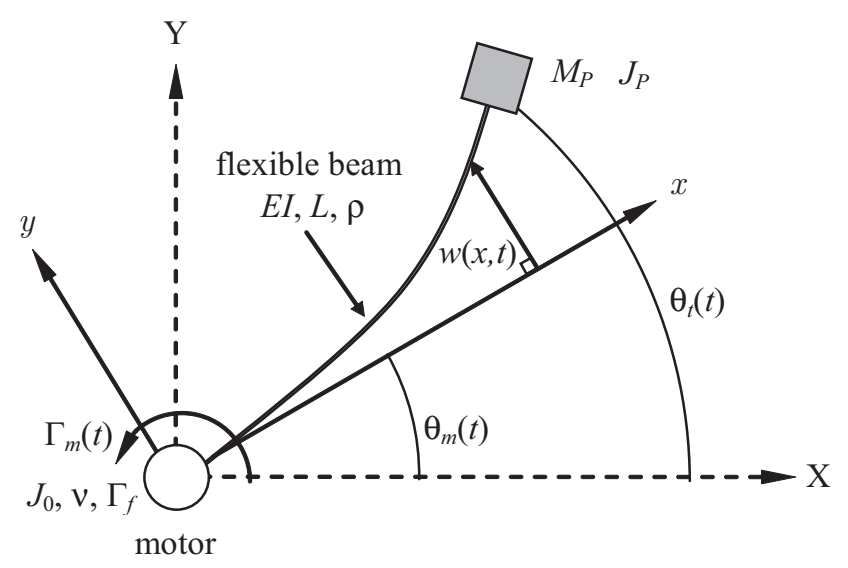

Fig. 2. Parametric representation of the flexible arm system

\section{A. Inner control loop}

The control law of the first loop is as follows:

$$
V(t)=\Gamma_{\text {coup }}(t) / n K+K_{p}\left(\theta_{m}^{*}(t)-\theta_{m}(t)\right)-K_{v} \dot{\theta}_{m}(t),
$$

where $\Gamma_{\text {coup }}(t) / n K$ (denoted as decoupling term) makes the design of the PD constants $\left(K_{p}, K_{v}\right)$ independent of the link dynamics. The substitution of (10) into (2) yields

$$
\begin{array}{r}
J_{0} \ddot{\theta}_{m}(t)+\left(n K K_{v}+\nu\right) \dot{\theta}_{m}(t)+n K K_{p} \theta_{m}(t) \\
=n K K_{p} \theta_{m}^{*}(t)+\Gamma_{f},
\end{array}
$$

which only depends on the motor dynamics. Thus, if $\Gamma_{f}$ is considered as a perturbation input, the motor angle can be expressed as follows

$$
\theta_{m}(s)=\frac{\theta_{m}^{*}(s)+\Gamma_{f}(s) /\left(n K K_{p}\right)}{s^{2}\left(J_{0} / n K K_{p}\right)+s\left(\left(n K K_{v}+\nu\right) /\left(n K K_{p}\right)\right)+1} .
$$

It must be noted that, in addition to the independence between $\theta_{m}(s)$ and the link dynamics, this inner loop is robust to Coulomb friction and to changes in the dynamic friction (with large values of $K_{p}$ ) [25]. Thus, if the tuning of the parameters of the PD controller $\left(K_{p}, K_{v}\right)$ is carried out to achieve a critically damped second-order system, Eq. (12) can be approximated by:

$$
\theta_{m}(s) \cong G_{m}(s) \theta_{m}^{*}(s)=\frac{\theta_{m}^{*}(s)}{(1+\alpha s)^{2}},
$$

where $\alpha$ is the constant time of the transfer function between $\theta_{m}$ and $\theta_{m}^{*}\left(G_{m}(s)\right)$. From Eqs. (12) and (13) the values of $K_{p}$ and $K_{v}$ are obtained.

$$
K_{p}=J_{0} / n K \alpha^{2} ; K_{v}=\left(2 J_{0}-v \alpha\right) / n K \alpha .
$$

\section{B. Outer control loop}

Let us denote $G_{1}(s)$ and $G_{2}(s)$ as the transfer functions $\theta_{t}(s) / \theta_{m}(s)$ and $\Gamma_{\text {coup }}(s) / \theta_{m}(s)$ respectively. The outer control loop imparts damping to link dynamics by using an IRC scheme for $G_{2}(s)$. As stated earlier, IRC is an easyto-tune, low-order controller that imparts substantial damping 


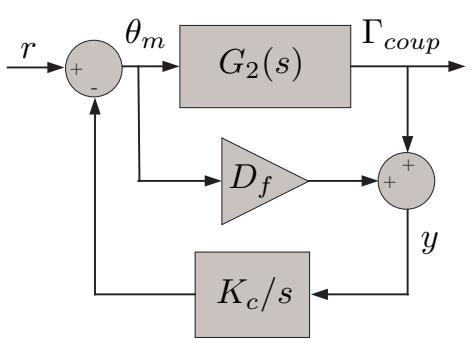

(a)

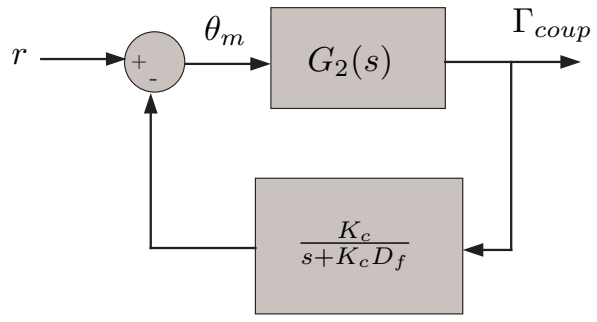

(b)

Fig. 3. (a) Schematic of the second control loop formed by IRC. (b) Modified IRC proposed in [26]

to multiple resonant modes of a flexible structure, without instability issues due to unmodeled system dynamics. If the IRC proposed in [22] is used (see Figure 3(a)), the next two problems appear. The first one is that the DC gain of $\theta_{t}(s) / \theta_{t}^{*}(s)$ is equal to zero. This can be proved by calculating the transfer function between $\theta_{m}(s)$ and $R(s)$ (from Figure $3(\mathrm{a}))$, which is

$$
\frac{\theta_{m}(s)}{R(s)}=\frac{s}{s+K_{c}\left(G_{2}(s)+D_{f}\right)} .
$$

Then, if $\theta_{m}=0 \mathrm{rad}$ in steady state and $w(L, t)=0$, the tip angle $\theta_{t}$ is also zero (see Eq. (9)). This problem is solved by using the alternative representation of IRC scheme, which is shown in Figure 3(b) [26]. The transfer function between $\theta_{m}(s)$ and $R(s)$ is as follows

$$
\frac{\theta_{m}(s)}{R(s)}=\frac{s+K_{c} D_{f}}{s+K_{c}\left(G_{2}(s)+D_{f}\right)},
$$

which has the same denominator as Eq. (15). In addition, the DC gain of Eq. (16) is equal to one because $G_{2}(0)=0$ due to the two zeros in the origin mentioned above. The second problem is derived from the impossibility of actuating directly on $\theta_{m}$. This is solved by using $\theta_{m}^{*}$ as error signal in the outer control loop. This requires of a dynamic inversion of $G_{m}(s)$, which is denoted by $\hat{G}_{m}^{-1}(s)=(s \hat{\alpha}+1)^{2} 1$. Then, the term $(s \beta+1)$ is included into the outer controller to make it implementable with relative order equal to 0 . The general control scheme is shown in Figure 4 and the transfer function of the overall system is as follows

$$
\frac{\theta_{t}(s)}{\theta_{t}^{*}(s)}=\frac{G_{m}(s) G_{1}(s)\left(s+K_{c} D_{f}\right)(s \beta+1)}{\beta s(s+1)+s+K_{c}\left(G_{m}(s) \hat{G}_{m}^{-1}(s) G_{2}(s)+D_{f}\right)} .
$$

Note that, if $\beta \rightarrow 0$ and $\alpha=\hat{\alpha}$, the design of IRC gains ( $D_{f}$ and $K_{c}$ ) is analogous to previous works (see [22] and [26]). Finally, it can be deduced that DC gain of $\theta_{t}(s) / \theta_{t}^{*}(s)$ is equal to one $\left(G_{1}(0)=1\right.$ and $\left.G_{2}(0)=0\right)$. Thus, the precise tip-positioning is achieved if the vibrations are canceled.

\section{Stability Analysis}

Due to the fact that $\theta_{t}(s) / \theta_{t}^{*}(s)$ and $\Gamma_{\text {coup }}(s) / \theta_{t}^{*}(s)$ have the same denominator and $G_{m}(s)$ is stable, the

\footnotetext{
${ }^{1}$ Ideally, $\hat{\alpha}=\alpha$, but we consider the case of an inexact cancellation of closed-loop motor dynamics
}

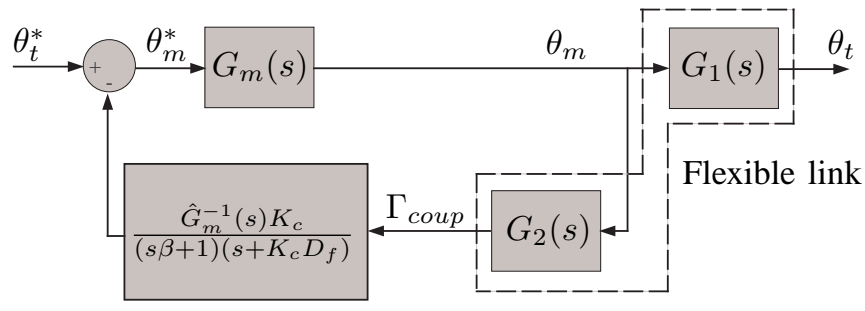

Fig. 4. General control scheme

stability of Eq. (17) can be demonstrated by considering the negative feedback interconnection of $G_{2}(s)$ and $K_{c} G_{m}(s) \hat{G}_{m}^{-1}(s) /\left((s \beta+1)\left(s+K_{c} D_{f}\right)\right)$. At this point, the results proved in [27], which are based on the feedback connection of systems with 'negative imaginary frequency response' (NIFR) ${ }^{2}$ are utilized. More precisely, the Theorem 5 in [27] states that, the positive feedback of two SISO systems $M(s)$ and $N(s)$, with $j\left[M(j \omega)-M(j \omega)^{*}\right] \geq 0$ and $j\left[N(j \omega)-N(j \omega)^{*}\right]>0$ for all $\omega \in(0, \infty)$, is internal stable if and only if $M(0) N(0)<1, M(\infty) N(\infty)=0$ and $N(\infty) \geq 0$.

As we have a negative feedback, we demonstrate that $-G_{2}(s)$ is NIFR, which is proved by using the passivity property of $G_{2}(s) / s$ deduced in [21]. Note that, $G_{2}(s) / s$ is passive if and only if it is positive real, i.e., $\operatorname{Re}\left[G_{2}(j \omega) / j \omega\right] \geq 0$, where $\operatorname{Re}[\star]$ is the real part of a complex number (see for example Section 4.7.2 of [28]). Then, if $\operatorname{Re}\left[G_{2}(j \omega) / j \omega\right] \geq 0$, it is easy to deduce the following equation:

$$
\begin{array}{r}
j\left[-G_{2}(j \omega)-\left(-G_{2}(j \omega)^{*}\right)\right]=\omega\left[\frac{G_{2}(j \omega)}{j \omega}-\frac{G_{2}(j \omega)^{*}}{-j \omega}\right] \\
=2 \omega \operatorname{Re}\left[\frac{G_{2}(j \omega)}{j \omega}\right] \geq 0 .
\end{array}
$$

Let us denote $C(s)$ as the following transfer function

$$
C(s)=\frac{K_{c}(s \hat{\alpha}+1)^{2}}{\left(s+K_{c} D_{f}\right)(s \alpha+1)^{2}(s \beta+1)} .
$$

The NIFR definition $j\left[C(j \omega)-C(j \omega)^{*}\right]>0, \forall \omega \in(0, \infty)$, is analogous to $\operatorname{Im}[C(j \omega)]<0, \forall \omega \in(0, \infty)$, where $\operatorname{Im}[\star]$ is

\footnotetext{
${ }^{2} \mathrm{~A}$ system $G(s)$ has negative imaginary frequency response when $j\left[G(j \omega)-G(j \omega)^{*}\right] \geq 0$ (or $\left.>0\right)$ for all $\omega \in(0, \infty)$
} 
the imaginary part of a complex number. If we substitute $s=$ $j \omega$ in Eq. (19) and multiply the numerator and denominator by the complex conjugate of the denominator, the result is as follows

$C(j \omega)=\frac{(1-j \beta \omega)(1-j \gamma \omega)\left[\left(1+\alpha \hat{\alpha} \omega^{2}\right)+j \omega(\hat{\alpha}-\alpha)\right]^{2}}{D_{f}\left(1+\beta^{2} \omega^{2}\right)\left(1+\gamma^{2} \omega^{2}\right)\left(1+\alpha^{2} \omega^{2}\right)^{2}}$,

in which $\gamma=1 / K_{c} D_{f}$. Furthermore, the next restriction obtained from the imaginary part of the numerator of $C(j \omega)$ can be written as:

$$
\begin{array}{r}
1-\frac{2(\hat{\alpha}-\alpha)}{\beta+\gamma}+ \\
\left(4 \alpha \hat{\alpha}-\hat{\alpha}^{2}-\alpha^{2}-\frac{2(\hat{\alpha}-\alpha)}{\beta+\gamma}(\alpha \hat{\alpha}-\beta \gamma)\right) \omega^{2}+ \\
\left(\alpha^{2} \hat{\alpha}^{2}+2 \frac{(\alpha+\hat{\alpha})}{\beta+\gamma} \alpha \hat{\alpha} \beta \gamma\right) \omega^{4}>0 .
\end{array}
$$

Note that the left side of Eq. (21) is a polynomial of the variable $\omega$. Let us denote the variable $z=\omega^{2}$ and the polynomial coefficients of $\omega^{4}, \omega^{2}$ and $\omega^{0}$ as $a_{2}, a_{1}$ and $a_{0}$ respectively. Thus, Eq. (21) can be rewritten as follows

$$
\phi(z)=a_{2} z^{2}+a_{1} z+a_{0}>0,
$$

This condition is fulfilled if $a_{0}>0(\phi(0)>0)$ and $a_{1}^{2}<4 a_{0} a_{2}$ (there is no sign change in $\phi(z)$ ). After further simplification, these restrictions can be expressed as:

$$
\begin{array}{r}
1-\frac{2(\hat{\alpha}-\alpha)}{\beta+\gamma}>0 \\
4\left(\alpha \hat{\alpha}-\beta^{2}\right)\left(\gamma^{2}-\alpha \hat{\alpha}\right)-(\hat{\alpha}-\alpha)^{2}(\beta+\gamma)^{2}- \\
4(\hat{\alpha}-\alpha)(\beta+\gamma)(\alpha \hat{\alpha}-\beta \gamma)>0
\end{array}
$$

Although this is not a explicit relation for obtaining the controller parameters, it is useful to analyze the affordable variation of $\alpha$ (due to motor parameters uncertainties) respect to its desired value $(\hat{\alpha})$, as it will be shown in next section.

As mentioned earlier, the DC gain of $G_{2}(s)$ is zero $\left(G_{2}(0)=0\right)$, and subsequently, $-G_{2}(0) C(0)=0<1$. Moreover, it can be deduced from Eq. (19) that $C(\infty)=0$, which implies that $G_{2}(\infty) C(\infty)=0$ and $C(\infty) \geq 0$. Therefore, according to Theorem 5 of [27] the closed loop system is internally stable. Note that, this stability property only depends on the controller parameters (Eq. (23)), i.e., the stability is independent of the link and payload parameters.

\section{EXPERIMENTAL RESULTS}

We set $\alpha=\hat{\alpha}=0.01$ and $\beta=0.001$, which allows us to neglect $G_{m}(s) \hat{G}_{m}^{-1}(s) /(s \beta+1)$ in the tuning of the IRC parameters and permits us to use the design strategy given in [22]. In addition, this value of $\alpha$ makes the transfer function $G_{m}(s)$ robust to Coulomb friction and does not saturate the DC motor. From Table I and Eq. (14), the values of $K_{p}$ and $K_{v}$ were 350.9 and 6.9. Identification for $G_{m}(s) G_{2}(s)$ with $M_{P}=0 \mathrm{~kg}$ and $J_{P}=0 \mathrm{kgm}^{2}$, which was used for IRC design, was carried out. The frequency response of $G_{m}(s) G_{2}(s)$ was obtained by applying a band limited random noise signal
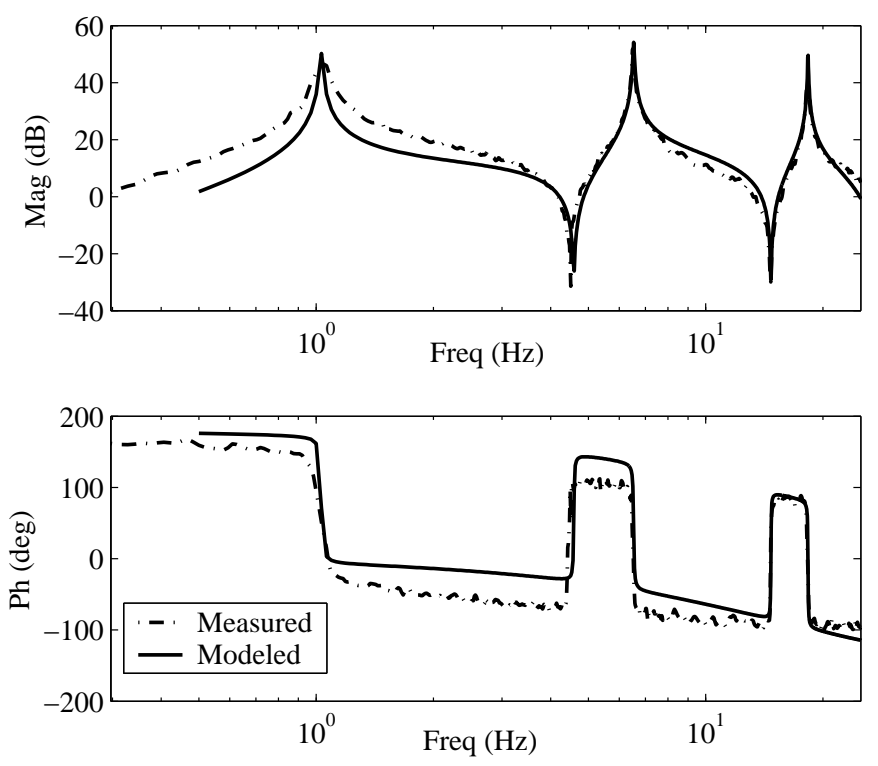

Fig. 5. Magnitude response of the measured (-.-) and modeled system (-) of $G_{m}(s) G_{2}(s)$

$(0.3$ to $25 \mathrm{~Hz})$ to the motor angle reference $\left(\theta_{m}^{*}\right)$. Both the input $\left(\theta_{m}^{*}\right)$ and the output $\left(\Gamma_{\text {coup }}\right)$ were recorded using the spectrum analyzer. Using a subspace-based system identification technique, an accurate model of the experimental system was obtained, [29]. Thus, the three first vibration modes are identified with sufficient accuracy to design an IRC and test the achievable damping in simulations. The identified transfer function is as following

$$
\begin{gathered}
G_{m}(s) G_{2}(s)=\frac{s^{2}}{(0.01 s+1)^{2}}\left(\frac{3.95}{s^{2}+0.07 s+43.5}+\right. \\
\left.+\frac{3.69}{s^{2}+0.25 s+1668}+\frac{2.68}{s^{2}+0.41 s+1.32 \cdot 10^{4}}\right)
\end{gathered}
$$

and the FRF of the measured and modeled system of $G_{m}(s) G_{2}(s)$ are plotted in Figure 5.

IRC parameters were tuned using the strategy given in [22]. First, a value $D_{f}>0$ was defined. Subsequently, $K_{c}$ was tuned to impart maximum damping to $\left(G_{2}(s)+D_{f}\right) / s$. It must be noted that, while selecting the value of $D_{f}$, there is a tradeoff between the time response of the system and the maximum damping that can be imparted. Small values of $D_{f}$ correspond to a controlled system with a dominant pole close to the origin. In this case, though the damping that can be imparted is large, the time response of the system (i.e., trajectory tracking) is very slow. If the value of $D_{f}$ is increased, this pole moves from the origin and can impart good damping. Thus, the performance of the system is improved to some extent by increasing $D_{f}$. Any increase in the value of $D_{f}$ beyond this point results in a poor damping performance. Therefore, after careful root locus analysis, tuning of $D_{f}$ should be realized together with $K_{c}$.

Due to practical considerations mentioned earlier, the proposed outer controller parameters were set to: $D_{f}=1$, $K_{c}=0.7(\gamma=1 / 0.7$ in Eq. (23)). This design considered the transfer function $G_{m}(s) G_{2}(s)$ of Eq. (24) and the maximum 

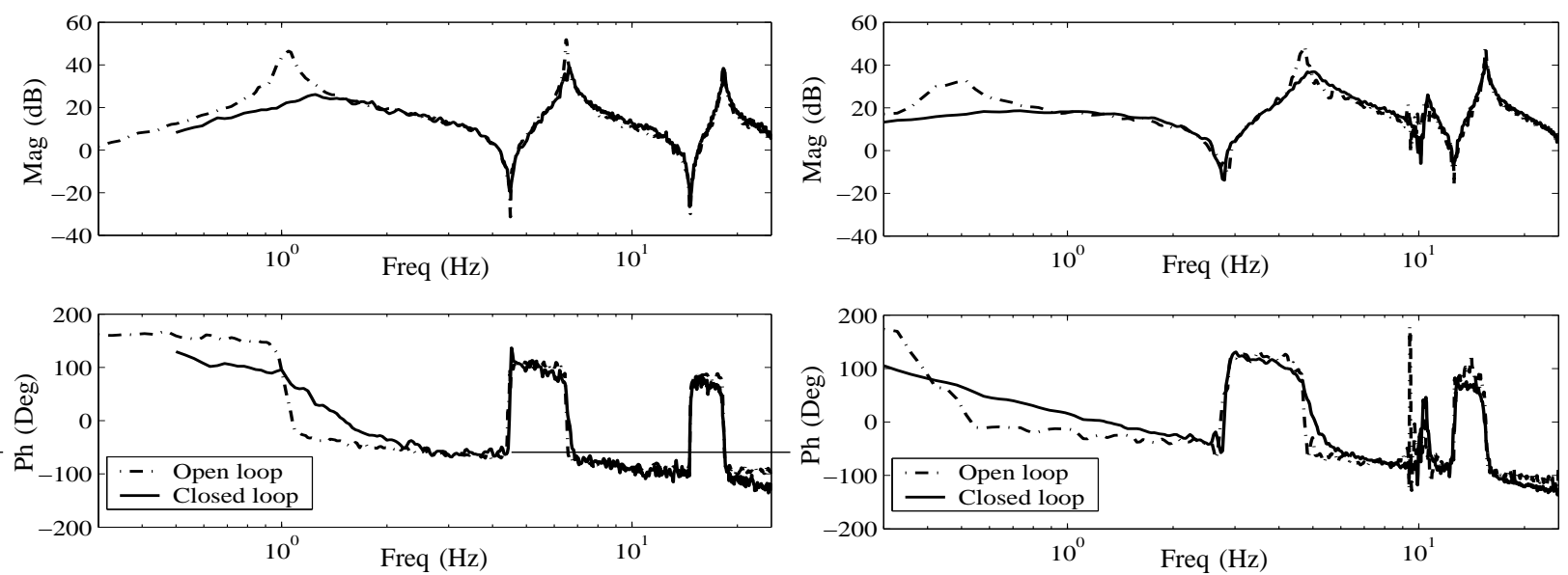

(a)

(b)

Fig. 6. Magnitude response of $G_{m}(s) G_{2}(s)$ (-.-) and the closed loop system between coupling torque and tip angle reference (一), (a) $M_{P}=0.0 \mathrm{~kg}$ and (b) $M_{P}=0.3 \mathrm{~kg}$. Note that in both the cases, there is substantial damping in the first two resonant modes.

variation of tip mass $(0.3 \mathrm{~kg}$ from Table I). In addition, it can be deduced from Eq. (23) that the controller system is stable if $\alpha \in(0.0012,0.056)$, which makes the controlled system robust to moderate uncertainties in $G_{m}(s)$. Figure 6 shows the FRF of the open loop $\left(G_{m}(s) G_{2}(s)\right)$ and closed loop for $M_{P}=0 \mathrm{~kg}$ (a) and $M_{P}=0.3 \mathrm{~kg}$ (b). The first three vibration modes are attenuated by $20.4 \mathrm{~dB}, 12.8 \mathrm{~dB}$ and $0.8 \mathrm{~dB}$ respectively for $M_{P}=0 \mathrm{~kg}$ and by $13.95 \mathrm{~dB}, 11.9 \mathrm{~dB}$ and $7.2 \mathrm{~dB}$ respectively for $M_{P}=0.3 \mathrm{~kg}$. It can be seen that the damping performance is quite robust to payload variations.

In order to illustrate the controller performance and show the slowdown in the time response of the system when the payload increases, experimental time responses were obtained. The used trajectory reference was a Bezier polynomial of fourth order with final value of $0.5 \mathrm{rad}$ and trajectory time of $1 \mathrm{~s}$. The three represented variables were the joint angle, the coupling torque measured at the base of the arm and the estimated tip angle, which was estimated using a full-order observer $^{3}$ whose inputs are the measured motor angle and the coupling torque. Fig. 7 shows the tip angle reference and the joint angle with IRC for $M_{P}=0 \mathrm{~kg}$ and $M_{P}=0.3 \mathrm{~kg}$ respectively. Figs. 8 (a) and (b) show the coupling torque without and with IRC for $M_{P}=0 \mathrm{~kg}$ and $M_{P}=0.3 \mathrm{~kg}$ respectively. It can be noted from Figs. 7 and 8 that the joint angle is accuracy positioned and the vibration is canceled for the two values of tip mass. Finally, Figs. 9(a) and (b) show the reference and the estimated tip angle of the open loop $\left(G_{m}(s) G_{2}(s)\right)$ and closed loop for $M_{P}=0 \mathrm{~kg}$ and $M_{P}=0.3 \mathrm{~kg}$ respectively. It can be observed that the system reaches the final position without vibration for the two values of tip mass. In addition, the settling time is bigger for $M_{P}=0.3 \mathrm{~kg}$. Finally, the steady state error in $\theta_{m}$ and $\theta_{t}$ is approximately equal to $0.02 \%$ and is due to the Coulomb friction. This can be reduced even more by higher proportional gain or by including an integral action in the inner loop.

\footnotetext{
${ }^{3}$ This full-order observer was only used to estimate the system output for comparison, and was not used for control purposes.
}

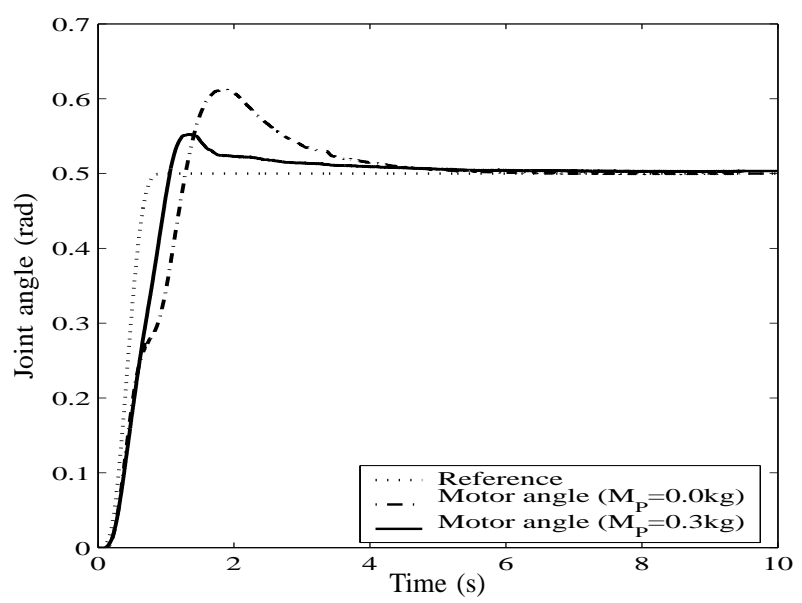

Fig. 7. Time response of the joint angle. Reference (...), $M_{P}=0.0 \mathrm{~kg}(-)$ and $M_{P}=0.3 \mathrm{~kg}(-.-)$

Therefore, it can be concluded from these experimental results that this novel control scheme is robust to big changes in the payload (by almost $90 \%$ when compared to the mass of the manipulator) because the damping is virtually unchanged and the stability is guaranteed. However, the time response is slowed down because the aforementioned pole associated to $D_{f}$ moves to the origin. This is due to the dependence of $D_{f}$ on the natural frequency of the first vibration mode, which decreases as the tip mass increases.

\section{A. Performance comparison of the most significant control schemes}

The election of a control strategy depends on the plant and particular application. This work has proposed a novel control approach that can be classified into the group of control strategies that use minimum phase dynamics to damp the vibration originated by link flexibility. These strategies are more convenient for making the controlled system robust to unmodeled high frequency dynamics (spillover) than those 


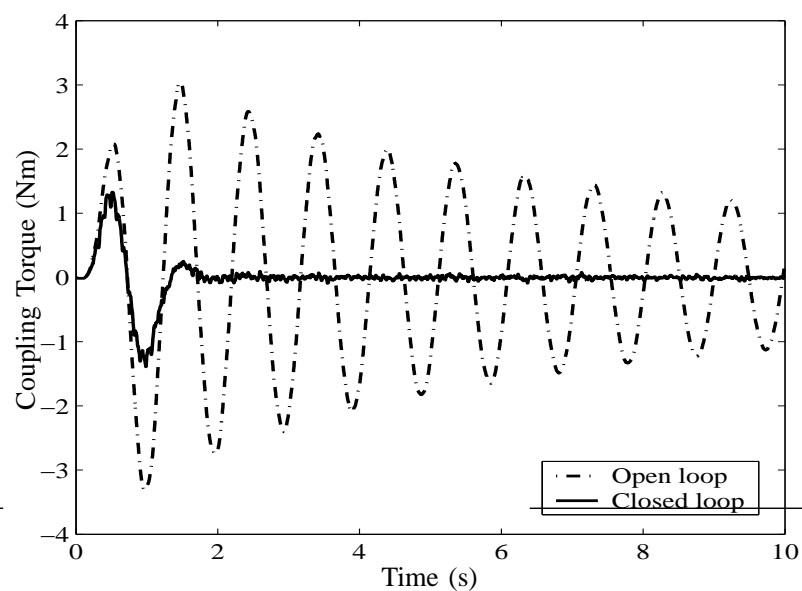

(a)

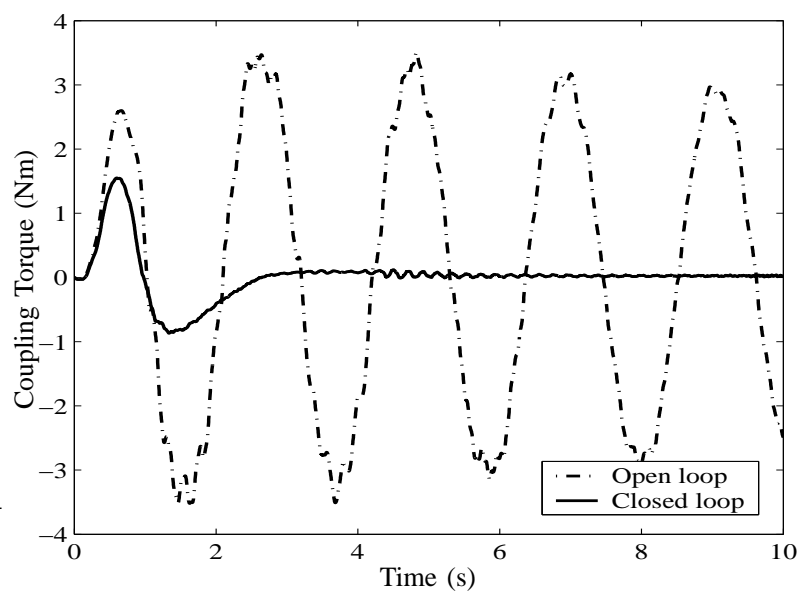

(b)

Fig. 8. Time response of the coupling torque in open loop (-.-) and closed loop (-), (a) $M_{P}=0.0 \mathrm{~kg}$ and (b) $M_{P}=0.3 \mathrm{~kg}$

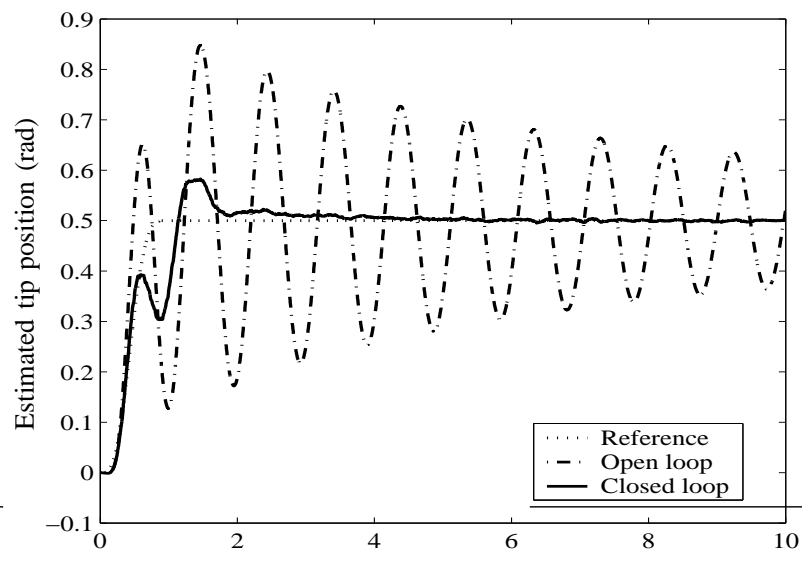

(a)

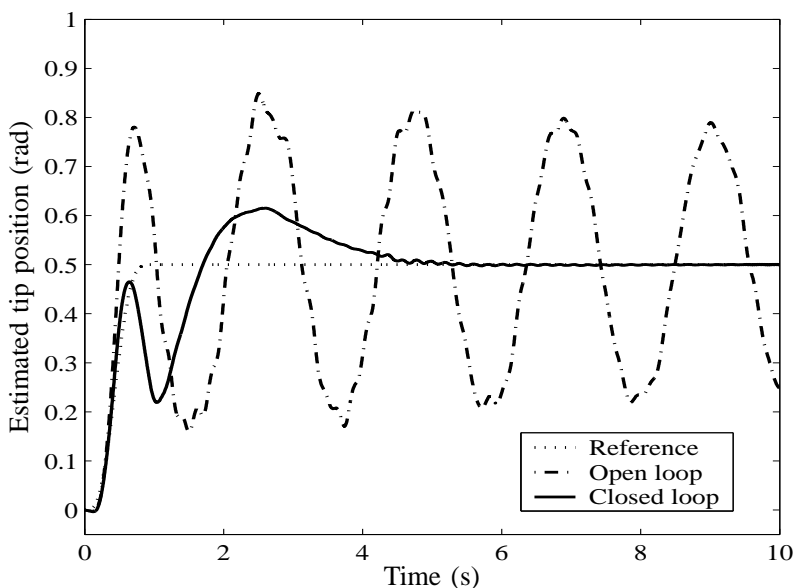

(b)

Fig. 9. Time response of the estimated tip angle obtained from experimental data, (a) $M_{P}=0.0 \mathrm{~kg}$ and (b) $M_{P}=0.3 \mathrm{~kg}$. Reference (..) and estimated tip position in open loop (-.-) and closed loop (-)

strategies that consider non-minimum phase dynamics (e.g., dynamics between tip position and motor torque). This is because the stability of the controlled system is simpler to obtain in practice.

The most significant references related to minimum phase systems, which have been cited in Introduction, are compared in this section. The precise tip positioning can be achieved by using an output related with tip position [16], [17], [20] or by canceling the vibration and positioning the joint [18], [19], [21]. Although the direct measurement of the tip presents the advantage of knowing the tracking error, these sensors might not be suitable in many industrial applications due to the need of visual contact between the tip and joint. This has motivated the use of others sensors such as strain gauges, which have been successfully used in practice [18], [19], [21]. In addition to the precise positioning under nominal conditions, these control techniques exhibit one or more of the following characteristics: i) robust to payload changes, ii) robust to joint frictions, iii) robust to external disturbances, iv) design simplicity and v) stability independent of payload parameters. In the next paragraphs, a performance comparison in terms of these characteristics is presented.

The reflected tip position and its generalization are used in [16], [17] respectively to accurately position the tip, which is achieved by a PD controller. Their main advantages are the robustness to payload changes and design simplicity. In addition, any external disturbance can be detected by the tip sensor. However, the passivity condition needed to guarantee the stability does not permit the use of an integral action. In addition, the PD gains are designed to achieve a good time response (i.e., imparted damping), which limits the value of these gains. Therefore, the controller is not robust to joint frictions. Moreover this passivity depends on the relationship between hub and link-plus-payload inertia, making the system stability highly dependent on payload.

To solve this problem, other control methodologies based 
on two control loops have been proposed. For example, in [18], [19] and [20] an inner loop to actively damp the link vibrations and an outer loop to achieve precise positioning are used. Sensors located in the link are used in [18] and [19] to impart damping. According to the experimental results showed in these works, the feedback of strain measured at the based of the link (inner loop) together a joint position control achieve precise positioning robust to joint frictions. In addition, we have checked in our experimental platform that this strategy is quite robust to payload changes. Moreover, the strain measured at the base can detect external disturbances, making these strategies robust to them. However, the design of the joint position control is complicated because the dynamics of the controlled system by the inner loop is considered. In addition, the stability demonstration depends of system parameters if an integral action is used. The joint angle is used in [20] to impart damping to the system by a resonant controller. Then, an integral controller of the tip position is used to guarantee precise tip positioning. This control scheme is robust to changes in the payload and joint frictions. However, the stability of overall system depends on the position control and the order of the inner controller increases with the number of considered vibration modes. In addition, the residual vibrations due to external disturbance are substantial if a reduction gear is employed, thus delivering poor disturbance rejection performance.

The strategy proposed in this work uses an inner loop to accurately position the joint angle and an outer loop to impart damping, which takes coupling torque as output (proportional to the strain measured at the base of the link) and joint angle as input. The joint control is designed without considering the link and can be made robust to joint frictions. The outer controller is based on the known inner loop dynamics and the transfer function between coupling torque and joint angle, which allows the detection of external disturbances in the link. Based on the internal stability property of the positive feedback of two systems with NIFR, the stability only depends on controller parameters. Thus, the stability of the overall system is independent of payload (i.e., robust to large payload changes). Finally, a simple and systematic tuning procedure for inner and outer controller exists and has been provided.

Table II shows a summary of the performance comparison expounded above. It can be seen that the contribution of the proposed methodology is to formulate a control law robust to joint frictions and external disturbances (like [18], [19]) combined with a simple design methodology, which allows us to make the stability of the overall system independent of payload parameters.

\section{CONCLUSION}

A new approach for the control of flexible manipulators was formulated and experimentally verified. This work follows the design methodology proposed in [21] to formulate a novel controller, which is based on a modification of the IRC scheme originally used in [22] to damp collocated smart structures. The resulting control scheme is internally stable if the given stability conditions are fulfilled, which are independent of payload parameters.

\begin{tabular}{|c|c|c|c|c|}
\hline $\begin{array}{c}\text { Robust to payload } \\
\text { changes }\end{array}$ & Yes & Yes & Yes & Yes \\
\hline $\begin{array}{c}\text { Robust to joint } \\
\text { frictions }\end{array}$ & No & Yes & Yes & Yes \\
\hline $\begin{array}{c}\text { Robust to external } \\
\text { disturbances }\end{array}$ & Yes & Yes & No & Yes \\
\hline Design simplicity & Yes & No & No & Yes \\
\hline $\begin{array}{c}\text { Independence } \\
\text { of stability }\end{array}$ & No & No & No & Yes \\
\hline
\end{tabular}

TABLE II

COMPARISON PERFORMANCE

The precise tip positioning is guaranteed by accurately positioning the joint angle and by damping the vibrations caused by the reference trajectory. Experimental results have been shown robustness to joint frictions, large changes in payload parameters and spill-over effects, which has resulted in a precise tip positioning. In addition, this new approach simplifies the positioning and damping control task to the tuning of two parameters $\left(K_{c}\right.$ and $\left.D_{f}\right)$ that can be adjusted intuitively without an exact knowledge of system dynamics. Therefore, the precise positioning and damping achieved by this simple control scheme makes it very suitable for many applications.

Future research endeavors will focus on multi-link flexible manipulators as well as parameter optimization.

\section{ACKNOWLEDGMENT}

The authors wish to thank the financial support provided by the Spanish Government Research Programme with the project DPI2006-13834 (MCyT).

The authors also would like to thank Dr. I. M. Díaz his help in Section III.

\section{REFERENCES}

[1] F. Wang and Y. Gao, Advanced studies of flexible robotic manipulators, modeling, design, control and applicattions. World Scientific, New Jersey (EUA), 2003.

[2] M. Benosman and G. Vey, "Control of flexible manipulators: A survey," Robotica, vol. 22, pp. 533-545, 2004.

[3] S. K. Dwivedy and P. Eberhard, "Dynamic analysis of flexible manipulators, a literature review," Mechanism and Machine Theory, vol. 41, no. 7, pp. 749-777, 2006.

[4] A. R. Fraser and R. W. Daniel, Perturbation techniques for flexible manipulators. Kluwer Academic Publisher, 1991.

[5] R. H. Canon and E. Schmitz, "Initial experiments on the end-point control of a flexible robot," International Journal on Robotics Research, vol. 3, no. 3, pp. 62-75, 1984.

[6] V. Etxebarria, A. Sanz, and I. Lizarraga, "Real-time experimental control of a flexible robotic manipulator using a composite approach," in Proceedings of the IEEE International Conference on Control Applications, September 2004, pp. 955-960.

[7] T. Kotnick, S. Yurkovich, and U. Ozguner, "Acceleration feedback control for a flexible manipulator arm," Journal of Robotic System, vol. 5, no. 3, pp. 181-196, 1998.

[8] A. De Luca, P. Lucibello, and G. Ulivi, "Inversion techniques of trajectory control of flexible robot arm," Journal of Robotic Systems, vol. 6 , no. 4, pp. 325-344, 1989.

[9] S. O. R. Moheimani and R. L. Clark, "Minimizing the truncation error in assumed modes models of structures," Transactions of the ASME, Journal of Vibration \& Acoustics, vol. 122, no. 3, pp. 332-335, July 2000. 
[10] T. C. Yang, J. C. S. Yang, and P. Kudva, "Load adaptive control of a single-link flexible manipulator," IEEE Transactions on Systems Management Cybernet, vol. 22, no. 1, pp. 85-91, 1992.

[11] D. Farruggio and L. Menini, "Two degrees of freedom h control of a flexible link," in Proceedings of the American Control Conference, June 2000, pp. 2280-2284.

[12] M. Karkoub and K. Tamma, "Modelling and mu-synthesis control of flexible manipulators," Computers and Structures, vol. 79, pp. 543-551, 2001.

[13] Y. P. Chen and H. T. Hsu, "Regulation and vibration control of an fembased single-link flexible-arm using sliding-mode theory," Journal of Vibration and Control, vol. 7, no. 5, pp. 741-752, 2001.

[14] Z. Su and K. A. Khorasani, "Neural-network-based controller for a single-link flexible manipulator using the inverse dynamics approach," IEEE Transactions on Industrial Electronics, vol. 48, no. 6, pp. 1074 1086, 2001.

[15] V. G. Moudgal, W. A. Kwong, K. M. Passino, and S. Yurkovich, "Fuzzy learning control for a flexible-link robot," IEEE Transactions on Fuzzy System, vol. 3, no. 2, pp. 467-488, 2002.

[16] D. Wang and M. Vidyasagar, "Passive control of a stiff flexible link," The International Journal of Robotics Research, vol. 11, no. 6, pp. 572-578, 1992.

[17] L. Liu and K. Yuan, "Noncollocated passivity-based pd control of a single-link flexible manipulator," Robotica, vol. 21, no. 2, pp. 117-135, 2003.

[18] Z. H. Luo, "Direct strain feedback control of flexible robot arms: new theoretical and experimental results," IEEE Transactions on Automatic Control, vol. 38, no. 11, pp. 1610-1622, 1993.

[19] Z. H. Luo and D. X. Feng, "Nonlinear torque control of a single-link flexible robot." Journal of Robotic System, vol. 16, no. 1, pp. 25-35, 1999

[20] I. A. Mahmood, S. O. R. Moheimani, and B. Bhikkaji, "Precise tip positioning of a flexible manipulator using resonant control," IEEE/ASME Transactions on Mechatronics, vol. 13, no. 2, pp. 180-186, 2008.

[21] E. Pereira, I. M. Díaz, J. J. Cela, and V. Feliu, "A new methodology for passivity based control of single-link flexible manipulator," in IEEE/ASME International Conference on Advanced Intelligent Mechatronics, 2007.

[22] S. S. Aphale, A. J. Fleming, and S. O. R. Moheimani, "Integral resonant control of collocated smart structures," Smart Mater. Struct., vol. 16, pp. 439-446, 2007.

[23] L. Meirovitch, Principles and techniques of Vibration. Englewood Cliffs, New Jersey: Prentice Hall, 1997.

[24] F. Bellezza, L. L. L, and G. Ulivi, "Exact modelling of the flexible slewing link," Proceedings of the IEEE International Conference on Robotics and Automation, vol. 1, pp. 734-804, May 1990.

[25] V. Feliu, D. S. Rattan, and H. B. Brown, "Control of flexible arms with friction in the joints," IEEE Transactions on Robotics and Automation, vol. 9, no. 4, pp. 467-475, 1993.

[26] E. Pereira, S. O. R. Moheimani, and S. S. Aphale, "Analog implementation of the integral resonant control scheme," Smart Materials and Structures, vol. 16, no. 6, pp. 1-6, 2008.

[27] A. Lanzon and I. R. Petersen, "Stability robustness of a feedback interconnection of systems with negative imaginary frequency response," IEEE Transactions of Automatic Control, vol. 53, no. 4, pp. 1042-1046, 2008

[28] J. J. Slotine and W. Li, Applied Nonlinear Control, N. J. Prentice Hall Inernational Editions, Englewood Cliffs, Ed., 1991.

[29] T. McKelvey, H. Akay, and L. Ljung, "Subspace based multivariable system identification from frequency response data," IEEE Transactions on Automatic Control, vol. 41, pp. 960-978, 1996.

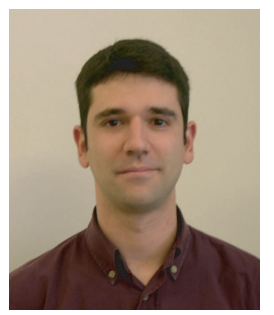

Emiliano Pereira received $\mathrm{Ph} . \mathrm{D}$. (with honors) degrees in Industrial Engineering from the Universidad de Castilla-La Mancha, Spain in 2009. He is working as research assistant in a research project from the Spanish Govement devoted to Monitoring and Control of Vibrations in Flexible Structures. His research involves active damping of vibrations in flexible structures and dynamic control of flexible robots.

Dr. Pereira is member of IEEE.

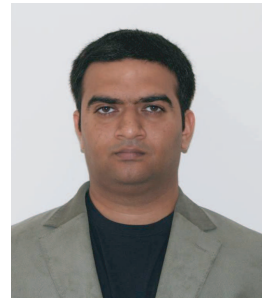

Sumeet S. Aphale received his Bachelors in Electrical Engineering from the University of Pune, India in 1999. He was a graduate student at the University of Wyoming, USA from 2000 onwards where he received his M.S.(May 2003) and Ph.D. (Aug 2005) both in Electrical Engineering. During this time, he was a member of the Hexapod Research Lab and worked on design and control of Gough-Stewart platform-type robotic manipulators. He was a member of the Australian Research Council's Center of Excellence for Complex Dynamic Systems and Control housed at the University of Newcastle, Australia from Oct 2005 - June 2008 and later moved to a senior research fellow appointment with the Center for Applied Dynamics Research at the University of Aberdeen, UK. Since June 2009, he has been appointed to the New Blood Lectureship position at the School of Engineering, University of Aberdeen, UK. His research interests include robot kinematics and control, vibration control applications as well as design and control of nanopositioning systems.

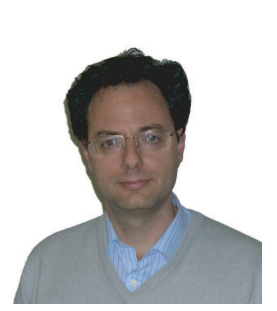

Vicente Feliu (M'88) received the M.S. degree (with honors) in industrial engineering and the Ph.D. degree from the Polytechnical University of Madrid, Spain in 1979 and 1982, respectively.

He was with the Electrical Engineering Department, Universidad Nacional de Educacin a Distancia, Spain, from 1980 to 1994 . He reached the position of Full Professor in 1990 and was Head of that Department from 1991 to 1994 . He has been Dean of the School of Industrial Engineering, Universidad de Castilla-La Mancha, Spain, from 1994 to 2008. He was a Fulbright Scholar, from 1987 to 1989 at the Robotics Institute, Carnegie Mellon University, Pittsburgh, PA. His research interests include multivariable and digital control systems, fractional dynamics and control, kinematic and dynamic control of rigid and flexible robots, mechatronics, automation of hydraulic canals, and computer vision for robots. He has written about 90 technical papers in international refereed journals and over 140 technical papers in international conferences. He was awarded with the prize to the best paper published in the Pattern Recognition Journal in 2001.

Dr. Feliu is a senior member of the IEEE (Institute of Electrical and Electronics Engineers) and member of IFAC (International Federation of Automatic Control)

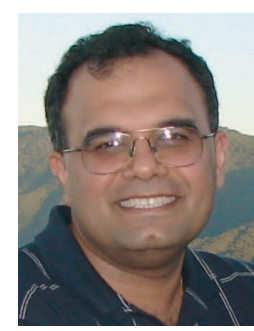

S. O. Reza Moheimani (SM00) received the Ph.D. degree in electrical engineering from the University of New South Wales at the Australian Defence Force Academy, Canberra, A.C.T., Australia, in 1996.

$\mathrm{He}$ is currently a Professor in the School of Electrical Engineering and Computer Science, University of Newcastle, Callaghan, N.S.W., Australia, where he holds an Australian Research Council Future Felowship. He is Associate Director of the Australian Research Council (ARC) Centre for Complex Dynamic Systems and Control, an Australian Government Centre of Excellence. He has held several visiting appointments at IBM Zurich Research Laboratory, Switzerland. He has published two books, several edited volumes, and more than 200 refereed articles in archival journals and conference proceedings. His current research interests include applications of control and estimation in nanoscale positioning systems for scanning probe microscopy, control of electrostatic microactuators in microelectromechanical systems (MEMS) and data storage systems.

Prof. Moheimani is a Fellow of the Institute of Physics, U.K. He is a recipient of the 2007 IEEE TRANSACTIONS ON CONTROL SYSTEMS TECHNOLOGY Outstanding Paper Award and the 2009 IEEE Control Systems Technology Award. He has served on the Editorial Board of a number of journals including the IEEE TRANSACTIONS ON CONTROL SYSTEMS TECHNOLOGY, and chaired several international conferences and workshops. 